\title{
Quantificação por NIR/MIR de Resina Poliuretânica em Misturas Binárias com Nitrocelulose Utilizadas em Tintas
}

\author{
Viviane Cristine Rodrigues \\ Instituto Tecnológico de Aeronáutica - ITA \\ Flint Group Tintas de Impressão LTDA
}

Rita de Cássia Lazzarini Dutra

Instituto Tecnológico de Aeronáutica - ITA

Divisão de Química - AQI, Instituto de Aeronáutica e Espaço - IAE

Milton Faria Diniz, Elizabeth da Costa Mattos

Divisão de Química - AQI, Instituto de Aeronáutica e Espaço - IAE

\begin{abstract}
Resumo: Foi elaborada uma nova metodologia nas regiões do infravermelho médio (MIR) e do infravermelho próximo (NIR), usando-se técnicas de Reflexão e/ou Transmissão para a determinação do teor de resina poliuretânica (PU) em misturas binárias com nitrocelulose (NC), utilizadas em formulações de tintas. A técnica de Reflexão Total Atenuada Universal (UATR) se mostrou útil na região MIR, para tal fim. Após a avaliação de diferentes bandas analíticas, de referência e de linhas de base, a banda relativa $\left(\mathrm{A}_{1541} / \mathrm{A}_{1645}\right)$ foi escolhida para a elaboração da curva de calibração, com erro de metodologia de 1,42\%. As técnicas de Transmissão e Reflexão (DRIFT) foram utilizadas na região NIR (banda relativa $\mathrm{A}_{5902} / \mathrm{A}_{5262}$ ) para validação, apresentando erros de metodologia de $1,42 \%$ e $1,60 \%$, respectivamente. Todos os erros relativos nas metodologias apresentaram-se dentro dos limites de precisão da análise quantitativa FT-IR, para as condições usadas $(\leq 2 \%)$. A análise de amostras teste confirmou a precisão das metodologias desenvolvidas, que também apresentam praticidade, baixo custo e tempo reduzido de análise.
\end{abstract}

Palavras-chave: Tintas, $P U, N C$, NIR, MIR, UATR, DRIFT e quantificação.

\section{Measurement by NIR/MIR of Polyurethane Resin in Binary Mixtures with Nitrocellulose Used in Paints Formulations}

\begin{abstract}
A new methodology was developed in the mid-infrared (MIR) and near infrared (NIR) regions by using techniques of Reflection and / or Transmission for the determination of polyurethane resin (PU) in binary mixtures with nitrocellulose (NC), used in paint formulations. The Universal Attenuated Total Reflection (UATR) technique proved useful in the MIR region for this purpose. From an evaluation of analytical bands and reference baselines, the relative band $\left(\mathrm{A}_{1541} / \mathrm{A}_{1645}\right)$ was selected for the calibration curve, whose error was $1.42 \%$. The Transmission and Reflection (DRIFT) techniques were used in the NIR region (relative band $\mathrm{A}_{5902} / \mathrm{A}_{5262}$ ) for validation, and both showed good precision with an error of methodology of 1.42 and $1.60 \%$ respectively. All relative errors are within the accuracy of FT-IR quantitative analysis for the conditions used $(\leq 2 \%)$. Analysis of test samples confirmed the accuracy of the methodologies, which also show practicality, low cost and reduced analysis time.
\end{abstract}

Keywords: Paints, $P U, N C$, NIR, MIR, UATR, DRIFT and measurement.

\section{Introdução}

Tintas representam sistemas multicomponentes complexos. Os componentes individuais, isto é, o ligante, o pigmento, e o solvente, também são de composição complexa. Desta forma, a análise minuciosa e completa de uma tinta exige uma grande experiência e o uso de um conjunto de modernas técnicas analíticas ${ }^{[1]}$.

Propriedades da tinta como dureza, flexibilidade, resistência à abrasão, resistência a álcalis, são governadas basicamente pela resina ${ }^{[2]}$.

É conhecido que a espectroscopia FT-IR pode ser favoravelmente utilizada para a análise de tintas e materiais relacionados ${ }^{[1-4]}$. Golton et al. ${ }^{[1]}$ reuniram um extenso trabalho onde demonstram diversas técnicas para caracterizar tintas, materiais relacionados, cura, durabilidade, problemas de revestimentos e controle de qualidade de tintas. Dentre as diversas técnicas analíticas, demonstram que a espectroscopia FT-IR pode ser utilizada em todo o campo de análise de tintas, com uso de acessórios como os de reflexão total atenuada (ATR), refletância difusa (DRIFT) e detecção fotoacústica (PAS).

Yang et al., em trabalho recente ${ }^{[3]}$, demonstraram que a análise qualitativa das amostras de tinta na área forense, pode ser realizada por FT-IR, porém evidências quantitativas ainda são difíceis, especialmente para polímeros sólidos ou curados. Neste trabalho, o 
poliestireno é quantificado por pirólise-cromatografia gasosa e espectrometria de massa (Pirólise-GC/MS), que constitui um acoplamento de técnicas, caro e pouco usual, apresentando menor disponibilidade em laboratórios, quando comparado à espectroscopia FT-IR na área de tintas.

Dutra et al. ${ }^{[4]}$ desenvolveram uma metodologia incluindo técnicas de FT-IR, que foi aplicada para a separação e caracterização dos principais componentes de tintas alquídicas, que pode ser aplicada, com algumas adaptações específicas e apropriadas, na caracterização de tintas comerciais e de composições similares.

Pode ser também observado, nos trabalhos citados e em geral, que são encontrados mais estudos sobre polímeros na região do infravermelho médio (MIR). Entretanto, a região do infravermelho próximo (NIR) tem um mérito quantitativo interessante, podendo em alguns casos, melhorar a precisão da metodologia desenvolvida para diferentes tipos de materiai ${ }^{[5-8]}$. Deste modo, as características da análise NIR devem ser abordadas para melhor entendimento da metodologia proposta neste trabalho.

É também conhecido que na região NIR são permitidas transições entre níveis de energia diferentes, originando os harmônicos ou sobretons, e as interações entre vibrações são possíveis, resultando em bandas de combinação. Os números de onda dos sobretons são, aproximadamente, múltiplos dos números de onda fundamentais e as de combinação correspondem à soma dos números de onda de seus componentes ${ }^{[5]}$. A maior energia da região NIR do que a do MIR excita sobretons e combinações de vibrações moleculares, fundamentais ou não, em materiais ao invés de excitar bandas fundamentais ${ }^{[5]}$.

Técnicas de análise de superfície podem também ser usadas em auxílio à técnica de Transmissão, tanto na região MIR quanto na região NIR, para uma avaliação qualitativa mais completa do material ${ }^{[9]}$. Por exemplo, DRIFT, onde as reflexões de superfície e do interior da amostra são medidas ${ }^{[10]}$ e Universal Reflexão Total Atenuada (UATR), de última geração, em que espécies de camada bem superficial são reveladas, têm encontrado destaque na análise de diferentes materiais, em comparação às técnicas de Transmissão[ ${ }^{[11]}$.

Todavia, estudos incluindo análise quantitativa por meio de técnicas de reflexão, são citados em menor número na literatura, devido às dificuldades encontradas, mas, por exemplo, em nossos laboratórios foi possível quantificar polímeros fluorados usados como coberturas poliméricas de materiais nitrados por ATR, com cristal de $\mathrm{Ge}$, o qual permite a avaliação de uma camada mais superficial do material, apresentando boa precisão, com uso de banda relativa, para correção de efeitos de variação de espessura ${ }^{[12]}$.

Entretanto, apesar dos trabalhos citados avaliarem diferentes polímeros, por diferentes técnicas, pode-se concluir que permitem algumas lacunas em relação à análise desses materiais, alguns usados também em tintas, como a aplicação de técnicas FT-IR de última geração para análises quantitativas e o uso da região NIR. Deste modo, com base neste fato e nas características de técnicas de análise FT-IR, disponíveis em nossos laboratórios, foi proposto o desenvolvimento de metodologia, com o uso de técnicas de reflexão FT-IR (comuns e de última geração), em ampla faixa espectral, que possam revelar e quantificar polímeros usados em tintas, em setores de ponta dessa indústria, visando contribuir, de forma significativa, para o aumento do potencial, em caracterização/quantificação, dos laboratórios de controle de qualidade e pesquisa das empresas do ramo das composições poliméricas, tornando-as mais competitivas nos mercados, nacional e internacional.

Portanto, especificamente, neste trabalho foi avaliada à aplicabilidade de técnicas FT-IR, tais como: UATR (de última geração), Transmissão (mais comum nos laboratórios) e DRIFT, explorando a região NIR, para a caracterização e quantificação de polímeros-base, usados em diferentes formulações industriais de tintas. Foi também avaliado se a metodologia desenvolvida para composições conhecidas, apresenta bons resultados para as composições poliméricas, similares, desconhecidas (amostras-teste).

\section{Materiais e Métodos}

\section{Amostras}

As amostras de resinas: Nitrocelulose (NC) e Resina Poliuretânica (PU), usadas neste trabalho, foram, gentilmente, cedidas pela empresa Flint Group. A PU analisada é de alto desempenho, contendo aproximadamente, $45 \%$ de polímero uretano, a NC, possui baixa nitração e aproximadamente $30 \%$ de sólidos, Ambas são fornecidas em solução de álcool etílico e acetato de etila.

\section{Equipamentos}

As amostras foram analisadas em espectrômetro FT-IR SPECTRUM ONE PERKINELMER com resolução: $4 \mathrm{~cm}^{-1}$, ganho 1, 7800 a $400 \mathrm{~cm}^{-1}$ (MIR e NIR) e 20 varreduras.

\section{Metodologia}

\section{Região MIR}

Foram preparadas amostras, em quantidade de massa igual a 2,0 g, completadas em balão volumétrico de $50 \mathrm{~mL}$ com álcool etílico.

Cinco amostras foram preparadas, mantendo-se a concentração de $\mathrm{NC}$ e variando a concentração de PU, com concentrações respectivas de resina NC/PU: 1,0/0,7; $1,0 / 1,4 ; 1,0 / 2,1 ; 1,0 / 2,8$ e $1,0 / 3,5$. Posteriormente, foram preparadas mais duas amostras, a fim de testar a curva de calibração, com concentrações de NC/PU, respectivas, de $1,0 / 1,0$ e $1,0 / 2,4$.

Com o auxílio de pipetas Pasteur, as soluções foram gotejadas ( $\sim 3$ gotas) no acessório UATR, em contato com a superfície do cristal de $\mathrm{ZnSe}$ com diamante, sem aplicação de torque, aguardando-se a evaporação total do solvente resultando na formação de uma película seca. Para cada amostra analisou-se cinco alíquotas. 


\section{Região NIR}

Foram preparadas amostras, em quantidade de massa igual a 5,0g, completadas em balão volumétrico de $50 \mathrm{~mL}$ com álcool etílico, com concentrações respectivas de resina NC/PU: $1,0 / 0,7 ; 1,0 / 1,4 ; 1,0 / 2,1 ; 1,0 / 2,8$ e 1,0/3,7. Posteriormente, duas amostras teste com concentrações de NC/PU, de 1,0/1,1 e 1,0/ 2,4.

As amostras foram colocadas em placa de Petri, aguardando a evaporação total dos solventes, analisaram-se cinco alíquotas da película seca formada.

Por Reflexão-UATR, as amostras foram analisadas colocando-as em contato com a superfície do cristal de ZnSe com diamante com aplicação de torque $(100 \mathrm{~N})$; por Transmissão, colocando-as no caminho do feixe; Reflexão-DRIFT: cortando as amostras em tamanho aproximado de $0,8 \times 0,8 \mathrm{~cm}$ e colocando-as em contato com a superfície do espelho de calibração.

\section{Fórmulas utilizadas nos cálculos por FT-IR}

Para cada amostra, foram analisadas cinco alíquotas e dentre os valores de absorvância, calculou-se a mediana $(\mu)$. O desvio médio padrão $\left(\hat{\sigma}_{\mu}\right)$ da absorvância mediana foi calculado segundo a Equação 1:

$$
\hat{\sigma}_{\mu}=\hat{\sigma} / \sqrt{n}
$$

Onde $\hat{\sigma}$ é o desvio padrão e $n$, o número de medidas. $\hat{\sigma}$ é dado pela Equação 2:

$$
\hat{\sigma}=K_{R} \cdot R
$$

$\mathrm{K}_{\mathrm{R}}$ é o coeficiente para cálculo do desvio padrão de uma faixa de valores (para 5 experimentos $\mathrm{K}_{\mathrm{p}} 0,430$ ) e $R$, a diferença entre o maior e menor valor de absorvância $\left(X_{n}-X_{1}\right)$. O erro relativo para cada amostra analisada foi determinado por meio da Equação 3:

$$
(\%)=\left(\hat{\sigma}_{\mu / \mu}\right) \times 100
$$

Para o erro da metodologia adotou-se o valor mediano dos erros encontrados. Os dados foram calculados de acordo com Hórak \& Vítek ${ }^{[13]}$, forma adotada em trabalhos anteriores do grupo, com sucesso, envolvendo análises quantitativas $\operatorname{IR}^{[7,8]}$.

\section{Resultados e Discussão}

\section{Região MIR - Espectros obtidos por Reflexão com acessório UATR}

\section{Caracterização das bandas principais das resinas NC e PU}

Os grupos funcionais mais característicos para a resina NC são os grupos nitro, estas absorções $\left(\mathrm{cm}^{-1}\right)$, estão em aproximadamente: $1640\left(\mathrm{NO}_{2}\right), 1270\left(\mathrm{NO}_{2}\right) \mathrm{e}$ $860(\mathrm{NO})^{[9]}$.

Tabela 1. Análise de bandas de referência (MIR-Reflexão-UATR).

\begin{tabular}{cccc}
\hline Banda Referência $\left(\mathbf{c m}^{-1}\right)$ & Atribuição & ERM (\%) & R \\
\hline 1375 & $\mathrm{CH}_{3}$ & 0,80 & 0,8602 \\
1454 & $\mathrm{CH}_{2} \mathrm{CH}_{3}$ & 1,03 & 0,8571 \\
1645 & $\mathrm{NO}_{2}$ & 1,09 & 0,9995 \\
1277 & $\mathrm{NO}_{2}$ & 1,18 & 0,9994 \\
2974 & $\mathrm{CH}_{2} \mathrm{CH}_{3}$ & 1,44 & 0,8766 \\
\hline
\end{tabular}

As absorções $\left(\mathrm{cm}^{-1}\right)$ características da resina PU estão em aproximadamente: $3330(\mathrm{~N}-\mathrm{H}), 1724$ (Amida $\mathrm{I}-\mathrm{C}=\mathrm{O}$ ), 1538 (Amida II-NH), 1250 (C-O-C=O poliéster) e 1100 (C-O-C poliéter) ${ }^{[14]}$.

\section{Escolha da banda analítica}

Pode-se observar na Figura 1, que o aumento da intensidade da banda em $1541 \mathrm{~cm}^{-1}$ está relacionado com o aumento do teor da resina PU no sistema binário, de acordo com a Lei de Lambert-Beer ${ }^{[9]}$, o que sugere ser possível determinar o teor de PU na mistura contendo $\mathrm{NC}$, por espectroscopia no infravermelho. Essa banda foi escolhida como analítica e foram analisadas duas possibilidades de linha base $1585 \mathrm{~cm}^{-1}$ a $1490 \mathrm{~cm}^{-1}$ e $1820 \mathrm{~cm}^{-1}$ a $1490 \mathrm{~cm}^{-1}$, para avaliação de maior precisão na medida de intensidade da banda. De acordo com o menor \% de Erro Relativo da Metodologia (ERM) 1,64\% e maior Coeficiente de Correlação (R) 0,9551, a linha base escolhida foi de $1585 \mathrm{~cm}^{-1}$ a $1490 \mathrm{~cm}^{-1}$.

Embora o estudo de linha base tenha apresentado bons resultados, uma melhor precisão para a metodologia pode ser buscada pela avaliação de banda de referência.

\section{Escolha da banda de referência}

Para eliminar o efeito da espessura, desde que não foi aplicado torque, pois a amostra está em solução e a análise é de acordo com o filme formado sobre o cristal, foram analisadas diversas possibilidades de bandas de referência.

As análises foram iniciadas, primeiramente, escolhendo-se os grupos $\mathrm{CH}_{2}$ e $\mathrm{CH}_{3}$ das cadeias poliméricas, e, posteriormente com o uso das bandas da resina de $\mathrm{NC}$, desde que não houve variação em sua

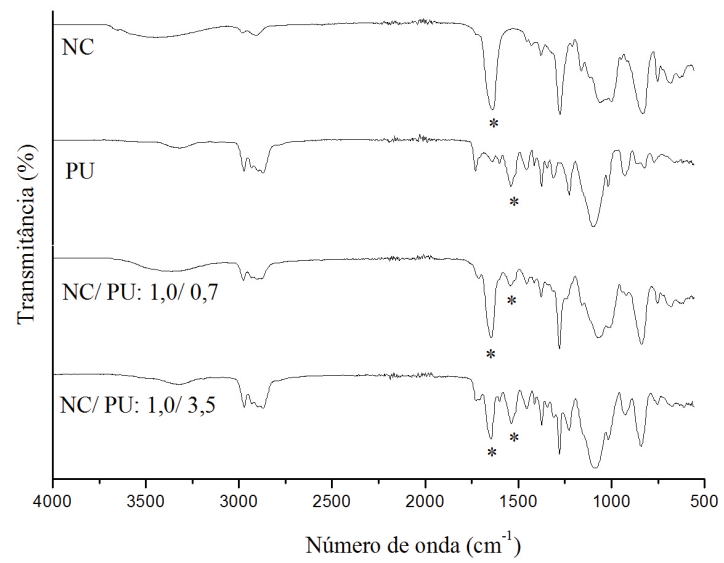

Figura 1. Espectros FT-IR (MIR-Reflexão-UATR) das resinas puras e misturas contendo menor e maior concentração de PU. 
concentração. A Tabela 1 mostra o ERM e R das bandas de referência analisadas.

A banda em $1645 \mathrm{~cm}^{-1}$ apresentou o terceiro menor erro e o melhor coeficiente de correlação. Considerada como melhor resultado, essa absorção foi adotada como banda de referência. A banda relativa (razão entre banda analítica e referência) apresentou menor ERM $(1,09)$ e maior R $(0,9995)$, demonstrando melhora na precisão da metodologia.

Foi elaborada uma curva de calibração com cinco pontos, onde $\mathrm{Y}$ é o valor mediano de $\mathrm{A}_{1541} / \mathrm{A}_{1645}$ e $\mathrm{X}$, o teor nominal de PU (\% p/p) em mistura binária e a seguinte equação da reta foi proposta: $Y=0,1730 X+0,0107$.

A fim de se verificar a eficácia do método foram sintetizadas duas novas amostras, com concentrações nominais de PU: 1,01 e 2,38 (\% p/p) para 1,0 NC. Os valores encontrados foram respectivamente: 0,91 e 2,35 (\% p/p). Os resultados mostraram-se próximos dos valores nominais, esses pontos foram inseridos na curva de calibração, apresentando novo $\mathrm{R}=0,9991$ e a seguinte equação da reta $\mathrm{Y}=0,1754 \mathrm{X}+0,0028$, demonstrado na Figura 2.

$\mathrm{O}$ erro relativo encontrado na metodologia UATR $(1,42 \%)$ está dentro dos limites de precisão do espectrômetro FT-IR $(\leq 2 \%)$.

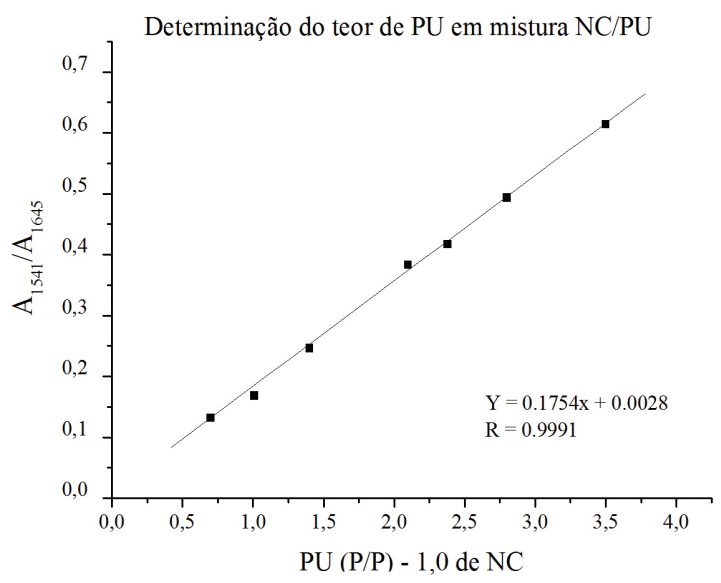

Figura 2. Curva de calibração FT-IR (MIR-Reflexão-UATR).

\section{Região NIR - Espectros obtidos por Reflexão com acessório UATR}

A proposta inicial foi utilizar o mesmo modo de obtenção de espectros da análise MIR (Reflexão-UATR). Os espectros apresentaram ruídos, não havendo absorção detectável do material nessas condições, provavelmente porque a amostra não apresentou bom contato com o cristal, o que impossibilita a análise quantitativa na região NIR desses materiais por UATR.

\section{Região NIR - Espectros obtidos por Transmissão}

Em busca de uma alternativa para análise qualitativa e quantitativa na região NIR, as mesmas amostras foram analisadas por Transmissão, colocando-se a película formada na direção do feixe.

Caracterização das bandas principais das resinas NC e PU - Análise de sobretons (ST) e bandas de combinação (BC)

A Tabela 2 inclui a atribuição provável das absorções das resinas puras. Algumas absorções não estão na região característica de sobretons (ST), sendo possível que as mesmas tratem-se de bandas de combinação (BC) de grupos característicos dos polímeros ${ }^{[5]}$.

\section{Escolha da banda analítica}

A princípio foram analisadas as absorções dos sobretons das bandas escolhidas na região MIR, que são as absorções: $4625 \mathrm{~cm}^{-1}\left(2^{\circ} \mathrm{ST} 1537 \mathrm{~cm}^{-1}\right)$ para banda analítica (PU) e $4835 \mathrm{~cm}^{-1}\left(2^{\circ} \mathrm{ST} 1636 \mathrm{~cm}^{-1}\right)$ para banda de referência $(\mathrm{NC})$.

Com o uso somente de banda analítica o erro da metodologia é alto $(9,80 \%)$, com o uso da banda relativa este erro diminui $(1,63 \%)$, mas em ambas as situações o coeficiente de correlação da equação é menor que 0,60.

Isso demonstra que não foi possível na região NIR utilizar os sobretons das bandas relativas utilizadas na região MIR, o que pode estar associado ao fato de que a $\mathrm{NC}$ e a PU apresentam absorções em regiões próximas de $4600 \mathrm{~cm}^{-1}$ e $4800 \mathrm{~cm}^{-1}$, possibilitando haver sobreposições.

Foram analisadas as absorções da resina PU e da NC onde não houvesse este tipo de interferência. Observou-se na Figura 3 que como banda analítica, era possível utilizar a absorção em $5902 \mathrm{~cm}^{-1}$, para a resina PU e como banda de referência, $5262 \mathrm{~cm}^{-1}$ para a resina NC. É possível

Tabela 2. Atribuição de grupos na região NIR: Sobretons (ST) e Bandas de Combinação (BC).

\begin{tabular}{|c|c|c|c|c|c|}
\hline \multicolumn{3}{|c|}{ Resina Nitrocelulose } & \multicolumn{3}{|c|}{ Resina Poliuretânica } \\
\hline $\mathrm{N}^{0}$ de onda $\left(\mathrm{cm}^{-1}\right)$ & Grupo Funcional & Atribuição Provável & $\mathrm{N}^{o}$ de onda $\left(\mathrm{cm}^{-1}\right)$ & Grupo Funcional & Atribuição Provável \\
\hline 4256 & $\mathrm{C}-\mathrm{O}-\mathrm{C}$ & $3^{\circ} \mathrm{ST} 1058$ & 4047 & & $\mathrm{BC}$ \\
\hline 4336 & & $\mathrm{BC}$ & 4332 & $\mathrm{C}-\mathrm{O}-\mathrm{C}$ & $3^{\circ} \mathrm{ST} 1095$ \\
\hline 4440 & & $\mathrm{BC}$ & 4412 & & $\mathrm{BC}$ \\
\hline 4640 & & $\mathrm{BC}$ & 4625 & Amida II-NH & $2^{\circ} \mathrm{ST} 1537$ \\
\hline 4835 & $\mathrm{NO}_{2}$ & $2^{\circ} \mathrm{ST} 1645$ & 4902 & $\mathrm{C}-\mathrm{O}-\mathrm{C}=\mathrm{O}$ & $3^{\circ} \mathrm{ST} 1224$ \\
\hline 5262 & $\mathrm{NO}_{2}$ & $3^{\circ} \mathrm{ST} 1274$ & 5206 & Amida $\mathrm{I}-\mathrm{C}=\mathrm{O}$ & $2^{\circ} \mathrm{ST} 1728$ \\
\hline 5838 & & $\mathrm{BC}$ & 5655 & & $\mathrm{BC}$ \\
\hline \multirow[t]{3}{*}{7032} & & $\mathrm{BC}$ & 5775 & & $\mathrm{BC}$ \\
\hline & & & 5902 & & $\mathrm{BC}$ \\
\hline & & & 5940 & & $\mathrm{BC}$ \\
\hline
\end{tabular}


Tabela 3. Dados FT-IR de erro relativo da metodologia.

\begin{tabular}{|c|c|c|c|c|}
\hline & Amostra NC: PU & Mediana $(\mu)$ & Desvio Médio Padrão & ERM \% \\
\hline \multirow{7}{*}{ 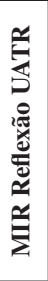 } & $1,0: 0,7$ & 0,132 & 0,001 & 0,91 \\
\hline & $1,0: 1,0$ & 0,168 & 0,002 & 1,42 \\
\hline & $1,0: 1,4$ & 0,246 & 0,007 & 2,92 \\
\hline & $1,0: 2,1$ & 0,383 & 0,006 & 1,46 \\
\hline & $1,0: 2,4$ & 0,417 & 0,020 & 4,82 \\
\hline & $1,0: 2,8$ & 0,493 & 0,004 & 0,82 \\
\hline & $1,0: 3,5$ & 0,614 & 0,007 & 1,09 \\
\hline \multirow{7}{*}{ 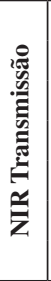 } & $1,0: 0,7$ & 0,989 & 0,058 & 5,85 \\
\hline & $1,0: 1,1$ & 1,365 & 0,019 & 1,42 \\
\hline & $1,0: 1,4$ & 1,573 & 0,043 & 2,76 \\
\hline & $1,0: 2,1$ & 1,941 & 0,024 & 1,23 \\
\hline & $1,0: 2,4$ & 2,297 & 0,048 & 2,10 \\
\hline & $1,0: 2,8$ & 2,485 & 0,014 & 0,54 \\
\hline & $1,0: 3,7$ & 2,715 & 0,026 & 0,96 \\
\hline \multirow{7}{*}{ 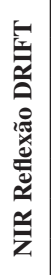 } & $1,0: 0,7$ & 0,962 & 0,033 & 3,42 \\
\hline & $1,0: 1,1$ & 1,309 & 0,015 & 1,15 \\
\hline & $1,0: 1,4$ & 1,511 & 0,017 & 1,16 \\
\hline & $1,0: 2,1$ & 2,089 & 0,032 & 1,51 \\
\hline & $1,0: 2,4$ & 2,435 & 0,039 & 1,60 \\
\hline & $1,0: 2,8$ & 2,605 & 0,075 & 2,88 \\
\hline & $1,0: 3,7$ & 3,051 & 0,062 & 2,04 \\
\hline
\end{tabular}

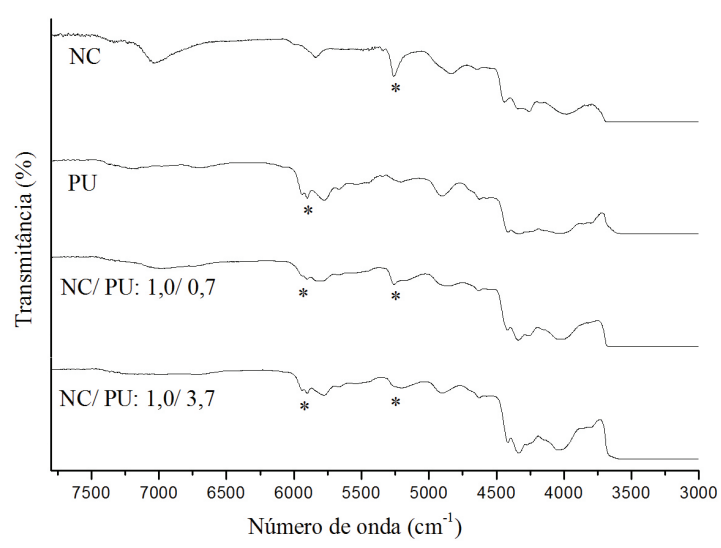

Figura 3. Espectros FT-IR (NIR-Transmissão) das resinas puras e misturas contendo menor e maior concentração de PU.

Tabela 4. Validação de metodologia MIR pelo uso da análise NIR.

\begin{tabular}{lcc}
\hline \multicolumn{1}{c}{ Comparação } & ERM (\%) & R \\
\hline MIR - Reflexão - UATR & 1,42 & 0,9991 \\
NIR - Transmissão & 1,42 & 0,9792 \\
NIR - Reflexão - DRIFT & 1,60 & 0,9906 \\
\hline
\end{tabular}

verificar que, com o aumento da concentração de PU, a absorção em $5902 \mathrm{~cm}^{-1}$ (PU) é intensificada.

Com o uso somente de banda analítica o ERM é alto $(9,66 \%)$ e o R é baixo $(0,6046)$, utilizando a banda relativa este erro diminui $(1,23 \%)$ e o coeficiente de correlação aumenta $(0,9837)$, demonstrando resultados melhores com uso da banda relativa, desde que não foi possível controlar a espessura da amostra. Foi elaborada uma curva de calibração com cinco pontos, e a seguinte equação da reta foi proposta: $Y=0,5879 X+0,6807$.

A fim de se verificar a eficácia do método foram sintetizadas duas novas amostras, com concentrações nominais de PU: 1,06 e 2,40 (\% p/p) para 1,0 NC. Os valores encontrados foram respectivamente: 1,16 e 2,75 (\% p/p). Os resultados mostraram-se próximos dos valores nominais, esses pontos foram inseridos na curva de calibração, apresentando novo $\mathrm{R}=0,9792$ e a seguinte equação da reta $\mathrm{Y}=0,5904 \mathrm{X}+0,7142$.

$\mathrm{O}$ erro relativo encontrados na metodologia NIR-Transmissão $(1,42 \%)$ está dentro dos limites de precisão do espectrômetro FT-IR $(\leq 2 \%)$.

\section{Região NIR - Espectros obtidos por Reflexão com acessório DRIFT}

A fim de se validar a metodologia por meio de análise qualitativa e quantitativa na região NIR por Reflexão, as mesmas amostras foram analisadas com uso do acessório DRIFT, colocando-se a película formada em contato com a superfície do espelho de calibração.

Os espectros obtidos por Reflexão não sofreram deslocamento e suas atribuições são as mesmas obtidas por Transmissão-NIR, os dados foram diretamente analisados com uso da banda relativa.

Foi elaborada uma curva de calibração com cinco pontos, e a seguinte equação da reta foi proposta: $\mathrm{Y}=0,7133 \mathrm{X}+0,5147$ e $\mathrm{R}=0,9948$. As mesmas amostras testes analisadas por Transmissão foram analisadas pelo método Reflexão-DRIFT, os valores encontrados foram respectivamente: 1,11 e 2,69 (\% p/p). Os dois pontos foram inseridos na curva de calibração, apresentando novo $R=0,9906$ e equação da reta $Y=0,7194 X+0,5382$. 
O erro relativo encontrado na metodologia ReflexãoDRIFT $(1,60 \%)$ está dentro dos limites de precisão do espectrômetro FT-IR $(\leq 2 \%)$.

Validação da metodologia MIR pelo uso da análise NIR

A Tabela 3 demonstra os valores de absorvância mediana e erro relativo das três metodologias aplicadas.

Na Tabela 4, é possível comparar os resultados obtidos por meio das três metodologias desenvolvidas no presente trabalho. Os erros relativos encontrados nas metodologias estão dentro dos limites de precisão do espectrômetro FT-IR $(\leq 2 \%)$, todos apresentaram altos valores de coeficiente de correlação R e na análise NIR, o melhor R encontrado foi na análise por Reflexão- DRIFT.

\section{Conclusões}

A técnica de Reflexão-UATR, na região MIR, bem como as técnicas de Transmissão e Reflexão-DRIFT, na região NIR, se mostraram úteis para quantificar o teor de Resina PU em sistemas binários com NC, demonstrando praticidade, baixo custo e tempo reduzido de análise.

Foram escolhidas as absorções $\left(\mathrm{cm}^{-1}\right)$ MIR e NIR respectivamente, em 1541 e 5902 como bandas analíticas para a resina PU, 1645 e 5262 como bandas de referência para a resina $\mathrm{NC}$, desde que não houve alteração na concentração desse polímero na mistura.

Os erros relativos encontrados na metodologia: MIR - Reflexão - UATR (1,42\%), NIR-Transmissão $(1,42 \%)$ e NIR - Reflexão - DRIFT (1,60\%) estão dentro dos limites de precisão do espectrômetro FT-IR $(\leq 2 \%)$, demonstrando que as metodologias são precisas, entretanto, o melhor $\mathrm{R}$ encontrado foi na análise por Reflexão - DRIFT. A análise de amostras-teste confirmou essa precisão.

A metodologia proposta apresentou análise FT-IR em ampla faixa espectral (NIR e MIR), fazendo uso de técnicas de Reflexão (UATR e DRIFT) e de Transmissão, demonstrando flexibilidade de aplicação, sendo que essas técnicas podem ser de grande utilidade na indústria e nos grandes centros de pesquisa, uma vez que ao determinar e quantificar o polímero base de uma formulação de tintas é possível prever as propriedades do produto final.

\section{Referências Bibliográficas}

1. Golton, W. C. - "Analysis of paints and related materials: Current techniques for solving coatings problems", ASTM International (1992). http://dx.doi.org/10.1520/STP1119-EB

2. Fazenda, J. M. R - "Tintas e vernizes: ciência e tecnologia", Edgard Blücher, São Paulo (2005). PMid:15704221.

3. Yang, S. H.; Shen, J. Y.; Chang, M. S. \& Wu, G. J. - Anal. Methods, 4, p.1989 (2012). http://dx.doi.org/10.1039/ C2AY05809J

4. Dutra, R. C. L.; Takahashi, M. F. K. \& Diniz, M. F.Polímeros, 12, p.273 (2002). http://dx.doi.org/10.1590/ S0104-14282002000400010

5. Goddu, R. F. \& Delker, D. A - "Spectra structure correlations for the near-infrared region", Analytical Chemistry (1960).

6. Araújo, S. C. \& Kawano, Y. - Polímeros, 11, p.213 (2001). http://dx.doi.org/10.1590/S0104-14282001000400011

7. Siqueira, S. H. S.; Dutra, R. C. L. \& Diniz, M. F. - Polímeros, 18, p.57 (2008).

8. Mattos, E. C.; Moreira, E. D.; Dutra, R. C. L.; Diniz, M. F.; Ribeiro, A. P. \& Iha, K. - Quím. Nova, 27, p.540 (2004). http://dx.doi.org/10.1590/S0100-40422004000400005

9. Smith, A. L. - "Applied Infrared Spectroscopy”, John Wiley \& Sons, New York (1979).

10. Pandey, G. C. \& Kulshreshtha, A. K. - Process Control Qual., 4, p.109 (1993).

11. Santos, R. P.; Oliveira, M. S.; Mattos, E. C.; Diniz, M. F. \& Dutra, R. C. L. - J. Aerosp. Technol. Manag., 5, p.65 (2013).

12. Mattos, E. C.; Diniz, M. F.; Nakamura, N. M.; Dutra, R. C. L. - J. Aerosp. Technol. Manag., 1, p.167 (2009). http:// dx.doi.org/10.5028/jatm.2009.0102167175

13. Hórak, M. \& Vítek, A. - "Interpretation And Processing Of Vibrational Spectra", John Wiley \& Sons, New York (1978).

14. Urbanski, J. \& Cameron, G. G. - "Handbook of analysis of synthetic polymers and plastics", John Wiley \& Sons, New York (1977). 